\title{
EPR Study of Gamma Irradiated Cholestanone Single Crystal
}

\author{
B. CALISKAN* \\ Department of Physics, Faculty of Arts and Sciences, Pamukkale University, 20070, Kinikli, Denizli, Turkey \\ (Received February 22, 2013; in final form September 28, 2013)

\begin{abstract}
Cholestanone $\left(\mathrm{C}_{27} \mathrm{H}_{46} \mathrm{O}\right)$ single crystals were irradiated with ${ }^{60} \mathrm{Co}-\gamma$ rays at room temperature. The spectra were recorded for different orientations of the crystal in the magnetic field at $120 \mathrm{~K}$ using the EPR technique. Radiation damage center was attributed to $\dot{\mathrm{C}} \mathrm{HCH}_{2} \mathrm{CH}_{2}$ radical. The principal values of the hyperfine coupling tensor of the unpaired electron with the protons and the principal values of the $g$ tensor were determined. The results were found to be in good agreement with the existing literature.
\end{abstract}

DOI: 10.12693 /APhysPolA.125.135

PACS 87.64.kh, 87.80.Lg, 61.72.Hh, 33.35.+r, 76.30.Rn, 32.10.Fn, 33.15.Pw, 71.18.+y, 76.30.-v, 61.82.Pv, 61.72.-y

\section{Introduction}

Cholestanone is a cholesterol derivative and cholesterol is also an important steroid alcohol. A steroid is a lipid characterized by a carbon skeleton with four fused rings. Rexroad and Gordy were the first to report on EPR study of free radicals in X-ray irradiated powdered cholesterol [1]. Cholesterol and its derivatives, such as cholesteryl benzoate [2], cholesteryl chloroformate [3], cholesteryl acetate [3], cholestanone, take a significant part in many biological mechanisms. Recently, the study of single crystal of cholesteryl derivatives by EPR techniques has yielded much information about the structure of free radicals formed in $\gamma$-irradiated single crystals of selected steroids.

However, $\gamma$-irradiated cholestanone single crystals were not investigated by EPR spectroscopy. Therefore, such a study on this compound was undertaken.

\section{Experimental}

Cholestanone powder was obtained from commercial sources. The single crystals were grown in the laboratory from its concentrated dimethyl sulfoxide solution by slow evaporation. The single crystals belong to space group $P 2_{1}$, with the cell dimensions of $a^{*}=34.120 \AA$, $b^{*}=19.967 \AA, c^{*}=11.036 \AA, \beta=98.33$ and $z=12$ [4]. The single crystals were irradiated at room temperature by a ${ }^{60} \mathrm{Co} \gamma$-ray source of $0.845 \mathrm{kGy} \mathrm{h}^{-1}$ for $142.5 \mathrm{~h}$. The EPR spectra were recorded with a Bruker EMX 081 EPR spectrometer using $3.994 \mathrm{~mW}$ microwave power. The modulation frequency of magnetic field was $100 \mathrm{kHz}$ and the modulation amplitude was $2 \mathrm{G}$. The low and high temperature measurements were carried out using a Bruker temperature control unit between 120 and $400 \mathrm{~K}$. The crystals were oriented in the cylindrical cavity by means of a goniometer capable of rotation about the axis perpendicular to the magnetic field. Spectra were recorded at intervals of $10^{\circ}$, at $120 \mathrm{~K}$.

*e-mail: bcaliskan@pau.edu.tr

\section{Results}

No EPR signal could be observed from the unirradiated cholestanone. After irradiation cholestanone by ${ }^{60} \mathrm{Co}-\gamma$ rays, at room temperature, EPR spectra could be obtained. The single crystals have been investigated between 120 and $400 \mathrm{~K}$. As the temperature increases, the spectra of the same axis and the angle demonstrate any change. The spectra have remained the same. For this reason, the spectra are independent of temperature. But, it is observed that the spectra depend on the magnetic field.

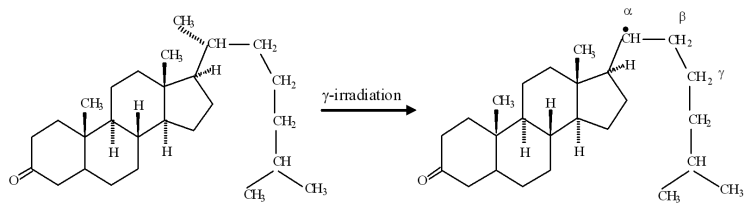

Fig. 1. The resonance form of cholestanone.

The resonance form of $\gamma$-irradiated cholestanone is shown in Fig. 1. It is determined that unpaired electron interacts with $\mathrm{H}_{\alpha}, \mathrm{H}_{\beta}$, and $\mathrm{H}_{\gamma}$ protons. Therefore these spectra belong to the $\dot{\mathrm{C}} \mathrm{HCH}_{2} \mathrm{CH}_{2}$ radical. The spectra exhibit a doublet (1:1) because of the $\mathrm{H}_{\alpha}$ proton directly attached to the $\mathrm{C}_{\alpha}$ carbon. Each of the lines (1:1) splits into the triplets (1:2:1) due to the two $\mathrm{H}_{\beta}$ protons, and then each of these lines splits into the triplet lines due to the two $\mathrm{H}_{\gamma}$ protons. The hyperfine interactions of the unpaired electron with $\mathrm{H}_{\alpha}, \mathrm{H}_{\beta}$, and $\mathrm{H}_{\gamma}$ protons are anisotropic. The spectroscopic splitting factor shows very small anisotropy. Also, obtained radical in this work is the same as that of Yigit et al. and Caliskan

TABLE I

Coupling constants values of simulated spectra.

\begin{tabular}{c|c|c|c|c}
\hline \hline & Fig. 2 & Fig. 4 & Fig. 6 & Fig. 8 \\
\hline$a_{\mathrm{CH}(\alpha)}[\mathrm{mT}]$ & 4.4 & 4.4 & 4.5 & 3.9 \\
$a_{\mathrm{CH}_{2}(\beta)}[\mathrm{mT}]$ & 2.3 & 2.1 & 2.017 & 2.1 \\
$a_{\mathrm{CH}_{2}(\gamma)}[\mathrm{mT}]$ & 0.705 & 0.654 & 0.631 & 0.6
\end{tabular}




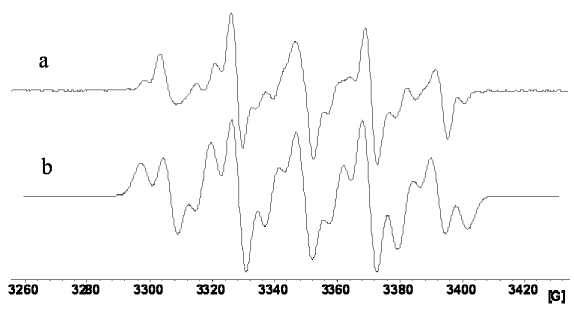

Fig. 2. (a) EPR spectrum of ${ }^{60} \mathrm{Co}-\gamma$ irradiated cholestanone single crystal at $120 \mathrm{~K}$ when the magnetic field is in the $a c$-plane at an angle of $30^{\circ}$ towards the axis. (b) Simulation of the spectrum; line width is $0.6 \mathrm{mT}$.

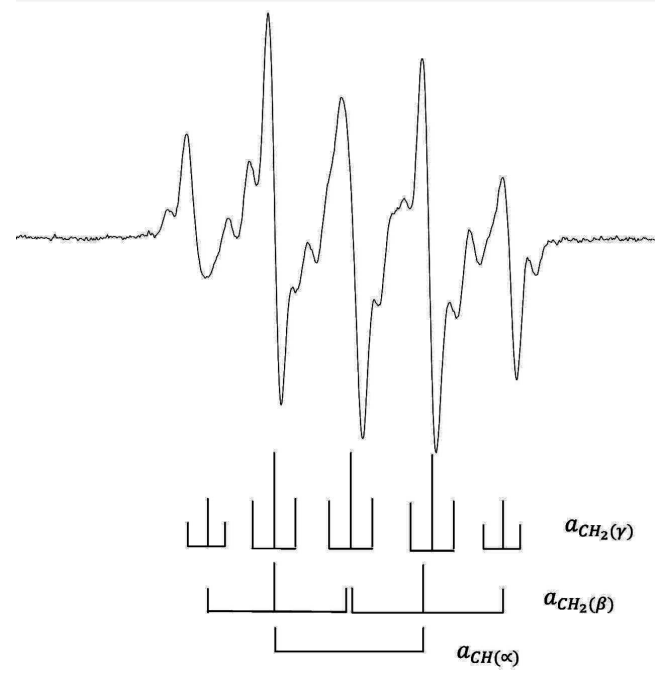

Fig. 3. The hyperfine structure splitting diagram of EPR spectrum of ${ }^{60} \mathrm{Co}-\gamma$ irradiated cholestanone single crystal at $120 \mathrm{~K}$ when the magnetic field is in the ac-plane at an angle of $30^{\circ}$ towards the axis.

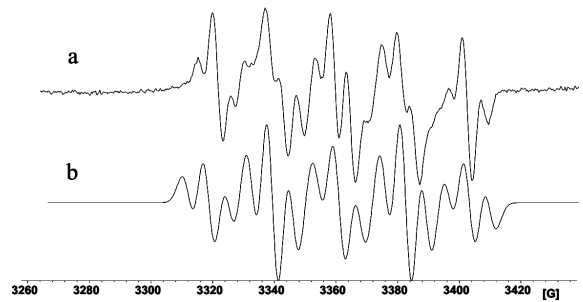

Fig. 4. (a) EPR spectrum of ${ }^{60} \mathrm{Co}-\gamma$ irradiated cholestanone single crystal at $120 \mathrm{~K}$ when the magnetic field is in the $a c$-plane at an angle of $70^{\circ}$ towards the axis. (b) Simulation of the spectrum; line width is $0.46 \mathrm{mT}$.

et al. $[2,3]$. But the hyperfine structure interaction values of $\mathrm{H}_{\alpha}$ proton of this study are different from the values of Yigit et al. and Caliskan et al. The hyperfine struc-

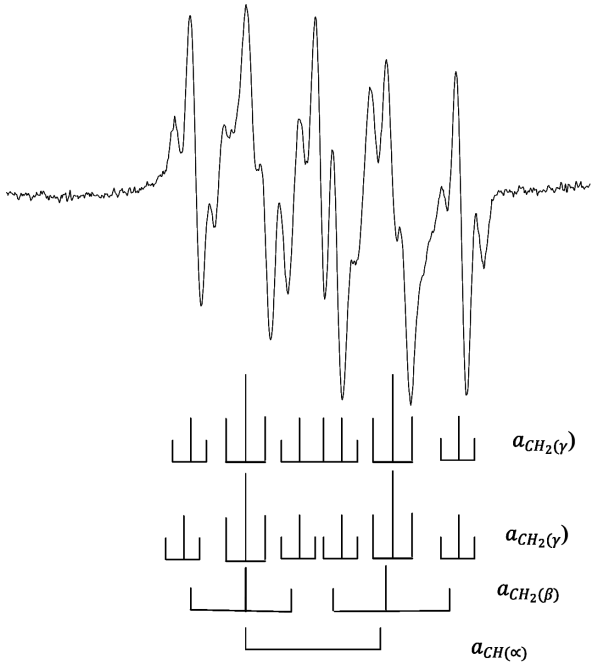

Fig. 5. The hyperfine structure splitting diagram of EPR spectrum of ${ }^{60} \mathrm{Co}-\gamma$ irradiated cholestanone single crystal at $120 \mathrm{~K}$ when the magnetic field is in the ac-plane at an angle of $70^{\circ}$ towards the axis.

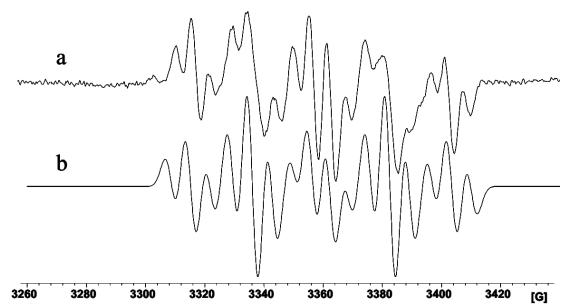

Fig. 6. (a) EPR spectrum of ${ }^{60} \mathrm{Co}-\gamma$ irradiated cholestanone single crystal at $120 \mathrm{~K}$ when the magnetic field is in the $a c$-plane at an angle of $90^{\circ}$ towards the axis. (b) Simulation of the spectrum; line width is $0.41 \mathrm{mT}$.

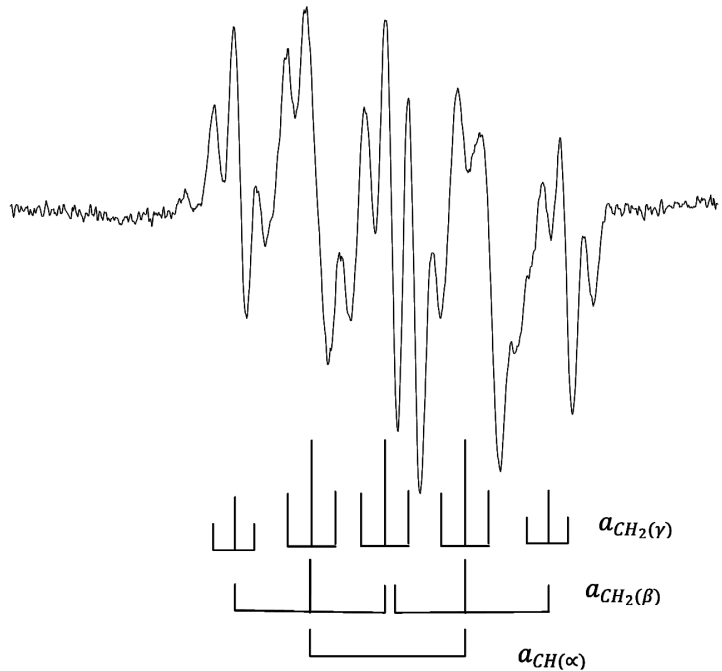

Fig. 7. The hyperfine structure splitting diagram of EPR spectrum of ${ }^{60} \mathrm{Co}-\gamma$ irradiated cholestanone single crystal at $120 \mathrm{~K}$ when the magnetic field is in the ac-plane at an angle of $90^{\circ}$ towards the axis. 


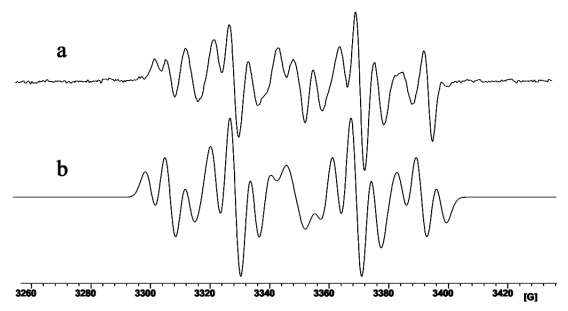

Fig. 8. (a) EPR spectrum of ${ }^{60} \mathrm{Co}-\gamma$ irradiated cholestanone single crystal at $300 \mathrm{~K}$ when the magnetic field is in the $b c$-plane at an angle of $60^{\circ}$ towards the axis. (b) Simulation of the spectrum; line width is $0.41 \mathrm{mT}$.

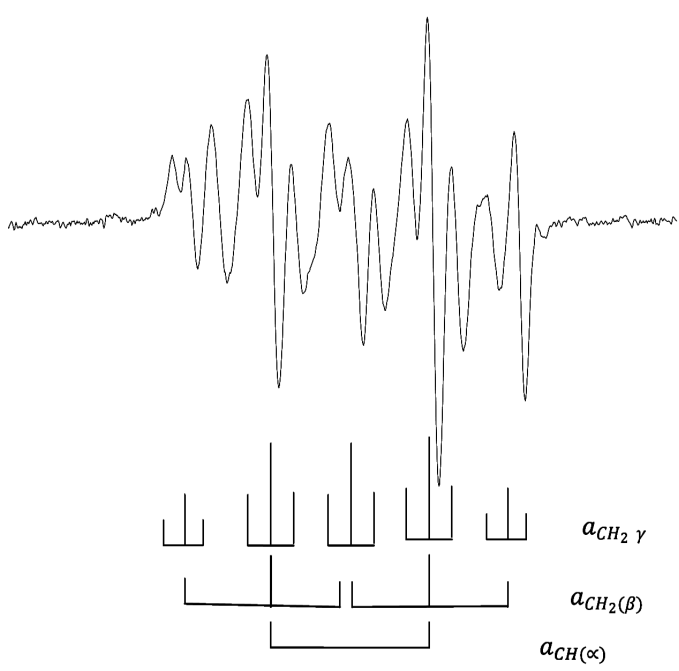

Fig. 9. The hyperfine structure splitting diagram of EPR spectrum of ${ }^{60} \mathrm{Co}-\gamma$ irradiated cholestanone single crystal at $300 \mathrm{~K}$ when the magnetic field is in the $b c$-plane at an angle of $60^{\circ}$ towards the axis.

ture interaction values of $\mathrm{H}_{\beta}$ protons are in the range of 1.961-2.431 $\mathrm{mT}$. The hyperfine structure interaction values of $\mathrm{H}_{\gamma}$ protons are in the range of $0.529-0.676 \mathrm{mT}$. These values for this radical agreed well with the literature values given for these radicals [2, 3, 5-14].

The simulations of the spectra are obtained with EPR simulation program. The simulation values of the hyper-

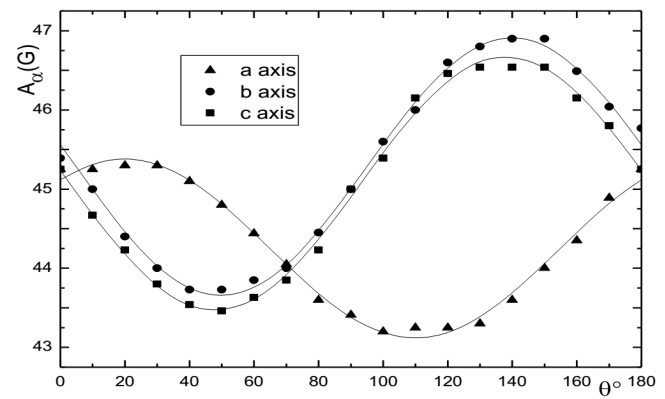

Fig. 10. The angular variation of the $A_{\mathrm{CH}(\alpha)}$ tensor for the $\dot{\mathrm{C}} \mathrm{HCH}_{2} \mathrm{CH}_{2}$ radical at $120 \mathrm{~K}$.

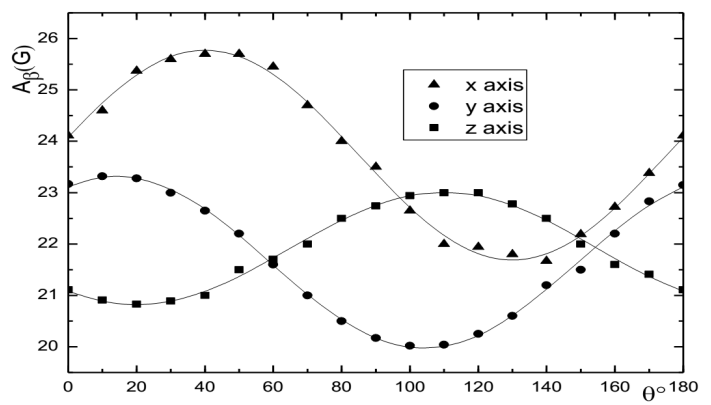

Fig. 11. The angular variation of the $A_{\mathrm{CH}_{2}(\beta)}$ tensor for the $\dot{\mathrm{C}} \mathrm{HCH}_{2} \mathrm{CH}_{2}$ radical at $120 \mathrm{~K}$.

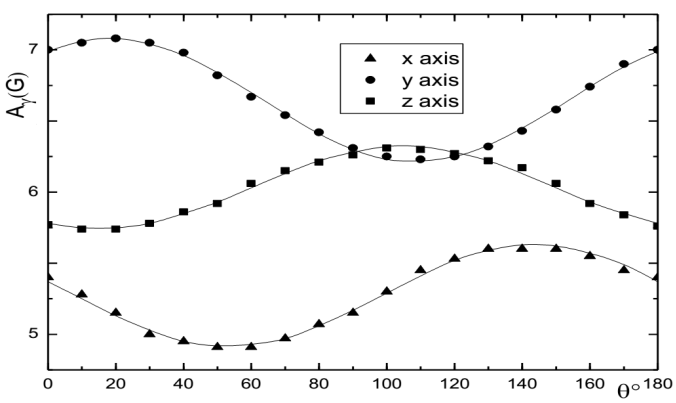

Fig. 12. The angular variation of the $A_{\mathrm{CH}_{2}(\gamma)}$ tensor for the $\dot{\mathrm{C}} \mathrm{HCH}_{2} \mathrm{CH}_{2}$ radical at $120 \mathrm{~K}$.

fine coupling constants of the spectra in Fig. 2, Fig. 4, Fig. 6, and Fig. 8 are given in Table I. The diagrams of the hyperfine structure splitting diagram of EPR spectra in Fig. 2, Fig. 4, Fig. 6, and Fig. 8 are given in Fig. 3, Fig. 5, Fig. 7, and Fig. 9, respectively.

The EPR parameters belonging to the $\dot{\mathrm{C}} \mathrm{HCH}_{2} \mathrm{CH}_{2}$ radical are included in Table II. The angular variations of $A$-values and the $g$-value of cholestanone at $120 \mathrm{~K}$ are shown in Figs. 10-13.

\section{Discussion}

The analysis of the EPR spectra and parameters pointed out that the free radical occurred by the removal

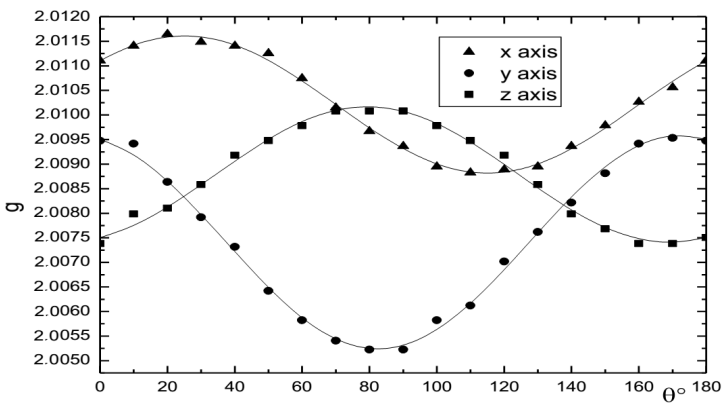

Fig. 13. The angular variation of the $g$-tensor for the $\dot{\mathrm{C}} \mathrm{HCH}_{2} \mathrm{CH}_{2}$ radical at $120 \mathrm{~K}$. 
TABLE II

The EPR parameters of the $\mathrm{CHCH}_{2} \mathrm{CH}_{2}$ radical observed in cholestanone at $120 \mathrm{~K}$. (Note: The errors are estimated to be \pm 0.000005 and $\pm 0.005 \mathrm{mT}$ for all the calculated $g$ and $A$-values, respectively.)

\begin{tabular}{c|c|c|c|c}
\hline \hline & Principal values & \multicolumn{3}{|c}{ Direction cosines } \\
\hline & $A_{x x}=4.754$ & 0.689248 & -0.229723 & 0.687143 \\
$A_{\mathrm{CH}(\alpha)}$ & $A_{y y}=4.402$ & -0.455251 & 0.600480 & 0.657396 \\
{$[\mathrm{mT}]$} & $A_{z z}=4.295$ & -0.563635 & -0.765931 & 0.309298 \\
& $a_{\mathrm{av}}=4.484$ & & & \\
\hline \multirow{3}{*}{$A_{\mathrm{CH}_{2}(\beta)}$} & $A_{x x}=2.431$ & 0.041000 & -0.950041 & -0.309422 \\
{$[\mathrm{mT}]$} & $A_{y y}=2.425$ & 0.704583 & 0.247066 & -0.665223 \\
& $A_{z z}=1.961$ & 0.708437 & -0.190739 & 0.679512 \\
& $a_{\mathrm{av}}=2.272$ & & & \\
\hline \multirow{3}{*}{$A_{\mathrm{CH}}(\gamma)$} & $A_{x x}=0.676$ & 0.924771 & 0.380272 & -0.013828 \\
{$[\mathrm{mT}]$} & $A_{y y}=0.590$ & -0.180486 & 0.470331 & 0.863837 \\
& $A_{z z}=0.529$ & 0.334997 & -0.796356 & 0.503582 \\
& $a_{\mathrm{av}}=0.598$ & & & \\
\hline \multirow{5}{*}{$g$} & $g_{x x}=2.009995$ & 0.964362 & 0.014410 & 0.264194 \\
& $g_{y y}=2.009388$ & 0.166723 & 0.742244 & -0.649059 \\
& $g_{z z}=2.007030$ & -0.205449 & 0.669975 & 0.713390
\end{tabular}

of the $\mathrm{CH}_{3}$ group from the carbon atom, unpaired electron was localized on the carbon atom in the substance. It was shown that the $\mathrm{CHCH}_{2} \mathrm{CH}_{2}$ radical was identified in the gamma-irradiated cholestanone single crystal. The hyperfine splitting of the $\alpha$-proton was anisotropic, its average value being $a_{\mathrm{CH}(\alpha)}=4.484 \mathrm{mT}$. The hyperfine splitting of two $\beta$-protons was slightly anisotropic, its average value being $a_{\mathrm{CH}_{2}(\beta)}=2.272 \mathrm{mT}$. The hyperfine splitting of two $\gamma$ was anisotropic, its average value being $a_{\mathrm{CH}_{2}(\gamma)}=0.598 \mathrm{mT}$. The $g$-value was also anisotropic and its average values being $g_{\text {av }}=2.008804$. The obtained measurement results implied that the radical structure in cholestanone was stable.

\section{Conclusions}

The radical produced from the cholestanone single crystal by ionized irradiation were investigated by EPR measurements. The $g$ values and hyperfine coupling constants of the unpaired electron with environmental protons were determined. Then, the $A$-values were verified by computer simulation. The radical was identified and radiation damage center was discussed using the spin Hamiltonian parameters obtained from the spectra.

\section{Acknowledgments}

My thanks to Professor Zeynel Kılıç for his support and encouragement.

\section{References}

[1] H.N. Rexroad, W. Gordy, Proc. Natl. Acad. Sci. USA 45, 256 (1959).

[2] B. Çalişkan, E. Aras, B. Aşik, M. Buyum, M. Birey, Radiat. Eff. Def. Solids 159, 1 (2004).

[3] B.T. Yiğit, A. Özmen, Ý. Ercan, M. Eken, M. Birey, Radiat. Eff. Def. Solids 158, 833 (2003).

[4] B.A. Haner, D.A. Norton, Acta Crystallogr. 20, 930 (1966).

[5] R.W. Fessenden, R.H. Schuler, J. Chem. Phys. 39, 2147 (1963).

[6] H. Fischer, Z. Naturf. 19a, 866 (1964).

[7] M.C.R. Symons, J. Chem. Soc., 277 (1959).

[8] M. Birey, Z. Naturf. 57a, 36 (2002).

[9] Ş. Osmanoğlu, M.H. Başkan, T. Güner, Z. Naturf. 57a, 909 (2002).

[10] P.B. Ayscough, C. Thomson, The Faraday Soc. Cont. 58, 1477 (1962).

[11] C. Heller, J. Chem. Phys. 36, 175 (1962).

[12] B.L. Bales, R.N. Schwartz, M.W. Hanna, J. Chem. Phys. 51, 1974 (1969).

[13] B. Smaller, M.S. Matheson, J. Chem. Phys. 28, 1169 (1958).

[14] W.T. Dixon, R.O.C. Norman, A.L. Buley, J. Chem. Soc., 3625 (1964). 